\title{
Using Ant Colony Optimization Clustering Algorithm with Power-Efficient Gathering in Sensor Information Systems Protocol
}

\author{
Talib M. Jawad ${ }^{*}$ and Noor A. Ali**1 \\ *Department Information and Communication Engineering, Al-Nahrain University, Baghdad-Iraq. \\ ***Department of Network, College of Information Engineering Al-Nahrain University, \\ Baghdad-Iraq. \\ ${ }^{1}$ E-mail: eng_noor_91@yahoo.com.
}

\begin{abstract}
Power-Efficient Gathering in Sensor Information Systems (PEGASIS) protocol is energy efficient protocols designed to prolong the lifetime of the network by reduction of energy consumption. In this paper a modification is proposed to the PEGASIS algorithm where sensor nodes are clustered in groups, clustering is done by Ant colony optimization algorithm, and each group is treated as PEGASIS. In addition, the proposed algorithm used rechargeable sensor nodes. Two parameters are searched to select chain leader: Euclidean distance of sensor node to the base station and residual energy of sensor node. Each cluster head data is transmitted directly to the base station. Simulation results showed the proposed algorithms improved in comparison with original PEGASIS.
\end{abstract}

Keywords: Wireless Sensor Network (WSN), PEAGSIS, Ant colony optimization (ACO), Sensor Node (SN), Base Station (BS).

\section{Introduction}

WSNs is one of popular technologies for the $21^{\text {st }}$ century. It is a sensing technology where very small devices called SNs distributed in a remote area to detect physical parameters, collect, treat data and forward information to users. They sense the ambient conditions and electronic signals generated from these sensed measurements that can be handled to describe some characteristic of the phenomena where SN located. These signals are transferred over radio waves to the BS [1-3]. Concentric Clustering [4] enhancement to PEGASIS scheme by dividing the sensor network into concentric clusters in order to consider the location of BS. In each concentric level, chain constructed between SNs by using Greedy algorithm and one node selected as cluster-head. Multiple-hops between clusterheads from the highest (farthest) cluster level to the lowest level near to the BS. Each SN is assigned to level according to signal strength to BS. Number of levels depends on number SNs and BS position. The protocol that proposed gave better results than PEGASIS protocol and reduces redundant data transmission. Simulation results showed that enhanced PEGASIS protocol is better than the PEGASIS and reduces redundant data transmission. Mobile sink improved energyefficient PEGASIS-based routing protocol (MIEEPB) [5] proposed which based on BS motion. The sensing area divided into four zones and nodes in each zone ordered into chain by Greedy algorithm. In order to elect the chain head need to find weight of each SN which is equal the residual energy for the node divided by its distance to the $\mathrm{BS}$. It is supposed that the BS has ineffective energy and it's able to move at fixed route from one zone to another, stop in each zone for specific time and collect data from sensors in their sites. PEGASIS with mobile sensor nodes (MPEGASIS) [6] where nodes change their position according to random waypoint mobility model, after moving, if a node doesn't find a close neighbor in its transmission range, it goes into sleep mode for a random period of time and then wakes up, this method is repeated until it discovers one in its transmission range. Gathered data is done by transfer information from sensor to another. Leader selection is based on residual energy and distance with BS for each sensor. In Sleep scheduled and tree-based clustering (SSTBC) algorithm [7] used the energy of SNs efficiently to prolong the network lifetime. SSTBC preserves energy by turning off the 
radio (entering sleep mode) of unnecessary nodes, which detect almost the same information, based on their location information to eliminate redundant data.

To reduce energy depletion SSTBC build minimum spanning tree with the root as the cluster head from active nodes in a cluster to forward data packets to BS.

Our proposed protocol adds feature to PEGASIS to support rechargeable sensors. In addition modified the topology PEGASIS by using ACO clustering algorithm.

\section{Description of the Proposed Algorithm}

The proposed approach based on clustering using swarm intelligent modified version of ACO used for grouping $\mathrm{N}$ nodes into $\mathrm{k}$ groups. The algorithm used to achieve the clustering problem to find optimal value of the objective function which is Euclidean distance $d_{i j}$ between the node and the center of the cluster. The smaller value of the objective function, means better clustering results. The formula of objective function expressed as follows [8][9]:

$\min F(w, C)=\sum_{j=1}^{k} \sum_{i=1}^{N} w_{i j} d_{i j}^{2}$

Where $\mathrm{w}$ is weight of node associated with cluster

$$
w_{i j}=\left\{\begin{array}{l}
1, \text { if node } i \text { belong to cluster } \\
\mathrm{S}_{j} \\
0, \text { otherwise }
\end{array}\right.
$$

Summation of weights for one node to all clusters must equal to one. Sensor node must belong to only one cluster at a time.

$$
\begin{aligned}
& \sum_{j=1}^{k} w_{i j}=1 \quad,(i=1,2,3, \ldots \ldots \ldots, N) \\
& \sum_{i=1}^{N} w_{i j} \geq 1 \quad,(j=1,2,3, \ldots \ldots \ldots, k)
\end{aligned}
$$

ACO procedure derived from real ant activities and used simulated ants called agents. ACO-PEGASIS can be represented in the following steps:

Step (0): Initialization of pheromone matrix to same small value, $\tau_{0}$ to ensure that probability will choose is not zero, number of ants $R$. The initial centers of clusters selected as shown in Fig. (1).

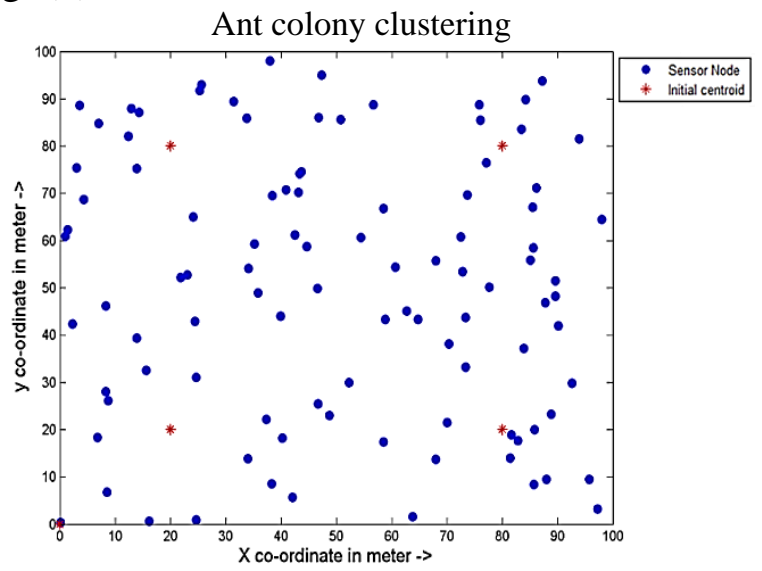

Fig.(1): Initial locations of centroids.

\section{Step (1): Solutions Construction}

Each agents construct solution of length $N$ which contain one element for each SN. Each element into solution represent the cluster number that SN belong to it. An agent build a solution for each SN and chooses one of clusters by applying state transition rule (STR). STR consist two type of strategies depending on $q_{0}$ which is predefined random number $\left(0<q_{0}<1\right)$ used to decide which strategy to follow in selecting appropriate cluster for each $\mathrm{SN}$.

If $\mathrm{q} \leq \mathrm{q}_{0}$ the ant choose the best option depend on heuristic information $\eta_{i j}$ and pheromone $\tau_{i j}$ as described in following equation:

$j=\underset{j \in k}{\arg \max }\left\{\tau_{i j} \cdot\left[\eta_{i j}\right]^{\beta}\right\}$

It means, the $\mathrm{SN}$ assigned to cluster $\mathrm{S}_{j}$ with chance $q_{0}$. This strategy called exploitation. Where $q$ is random number uniformly scattered in interval $[0,1], \beta$ is the parameter which determines the relative influence of heuristic information and $\eta_{i j}$ is heuristic information value which is inverse of Euclidean distance $d_{i j}$ between node $i$ and the center of cluster $S_{j}$ as shown in following equation:

$\eta_{i j}=\frac{1}{d_{i j}}$ 
If $\mathrm{q}>\mathrm{q}_{0}$ the ant select cluster $\mathrm{S}_{j}$ with probability $\left(1-q_{0}\right)$ according to roulette rule probability, this strategy called Exploration.

$P_{i j}=\frac{\tau_{i j}\left[\eta_{i j}\right]^{\beta}}{\sum_{m=1}^{k} \tau_{i m}\left[\eta_{i m}\right]^{\beta}}$

When every $\mathrm{SN}$ assigned to elected cluster. The agent has a complete solution building. In Fig.(2) an example of solution string generated by one agent for 8 SNs and 3 clusters. It shows that first node assign to cluster 2 and second $\mathrm{SN}$ assigned to cluster 1 and so on.

\begin{tabular}{|c|c|c|c|c|c|c|c|}
\hline $\begin{array}{l}\text { Z } \\
\text { oु } \\
\text { Z }\end{array}$ & $\begin{array}{l}\text { N } \\
\text { on } \\
\text { ż }\end{array}$ & 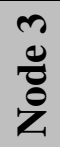 & $\begin{array}{l}\nabla \\
\ddot{g} \\
\text { Z } \\
Z\end{array}$ & $\begin{array}{l}\text { in } \\
\text { हु } \\
\text { Z }\end{array}$ & $\begin{array}{l}0 \\
0 \\
\tilde{n} \\
z \\
z\end{array}$ & $\begin{array}{l}r \\
0 \\
\tilde{z} \\
z\end{array}$ & $\begin{array}{l}\infty \\
0 \\
\ddot{Z} \\
Z \\
Z\end{array}$ \\
\hline 2 & 1 & 3 & 2 & 2 & 3 & 2 & 1 \\
\hline
\end{tabular}

Fig.(2): Example of solution string.

After solution constructed the weight of node $i$ associated with cluster $\mathrm{S}_{j}$ is calculated using Eq. (2). Then center of each cluster $S_{j}$ is calculated by the following:

$C_{j}=\frac{\sum_{i=1}^{N} w_{i j} x_{i}}{\sum_{i=1}^{N} w_{i j}},(j=1,2,3, \ldots \ldots, k)$

\section{Step (2): Crossover operator}

It is generates new solutions from recombining the current solutions hopefully get better features from parent solutions. ACO use crossover in order to improve solutions generated by ants. First the solutions sorted in ascending order according to their objective function values then crossover operator procedure performed on top $L(L<R)$ solutions with lowest objective function values.

There are several categories of crossover, one of them parameterized uniform crossover. It is generate random number between 0 and 1 for each element into solution crossover probability $p_{\text {crossover. }}$ Fig. ( $(\boldsymbol{)})$ Shows example of uniform crossover where eight RN generated and the crossover probability $p_{\text {crossover }}=0.7$.

\begin{tabular}{|c|c|c|c|c|c|c|c|c|c|c|}
\hline Parent 1 & 1 & 3 & 1 & 2 & 1 & 3 & 2 & 1 \\
\hline Parent 2 & 2 & 1 & 2 & 1 & 2 & 3 & 1 & 3 \\
\hline $\begin{array}{c}\text { Random } \\
\text { Number }\end{array}$ & 0.231 & 0.742 & 0.655 & 0.198 & 0.001 & 0.127 & 0.874 & 0.436 \\
\hline $\begin{array}{c}\text { Crossover } \\
\text { probability }\end{array}$ & $<0.7$ & $>0.7$ & $<0.7$ & $<0.7$ & $<0.7$ & $<0.7$ & $>0.7$ & $<0.7$ \\
\hline $\begin{array}{c}\text { New Solution } \\
\text { String 1 }\end{array}$ & 1 & 1 & 1 & 2 & 1 & 3 & 1 & 1 \\
\hline $\begin{array}{c}\text { New Solution } \\
\text { String 2 }\end{array}$ & 2 & 3 & 2 & 1 & 2 & 3 & 2 & 3 \\
\hline
\end{tabular}

Fig.( ('): Uniform crossover operator.

If the value of random number corresponding to element is less than the crossover probability, the elements from the first parent are selected, and the elements from the second parent are chosen other otherwise. After performing crossover the objective function calculated to new generated solutions using Eq. (1). These solutions accepted only if there is improvement in the objective function values, means If new calculated objective function value is less than first calculated objective function value new generated solution replaced with previous one otherwise the new solution is ignored. Best-so-far solution is selected from new solutions with lowest objective function value.

\section{Step (3): The global pheromone trail modernizing rule}

Only the ant with best-so-far solution is allowed to add pheromone after each iteration, the pheromone trial modernized by both adding pheromone between $\mathrm{SN}$ and selected cluster and evaporating the pheromone to other clusters. Thus pheromone trial modernized using following equation:

$\tau_{i j}(t+1)=(1-\rho) \tau_{i j}(t)+\rho \Delta \tau_{i j}^{b s}$

Where $\rho$ is the persistence rate of trail that lies between $[0,1],(1-\rho)$ the evaporation rate.

$\Delta \tau_{i j}^{b s}=\left\{\begin{array}{c}1 / F_{b s}, \text { if cluster } j \text { is assigned to } \\ i \text { th element of best solution } \\ 0 \quad, \text { otherwise }\end{array}\right.$ 
Where $F_{b s}$ is fitness of best-so-far solution. The basic steps of algorithm simplified by flowchart in Fig. (4).

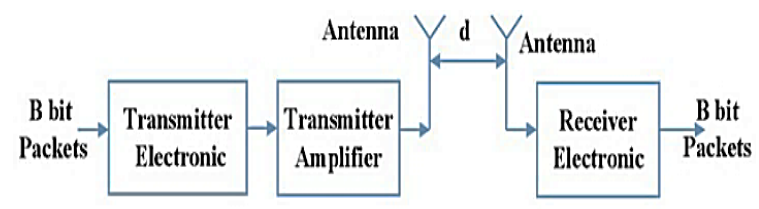

Fig.(4): First order radio mode.

\section{Step (4): Chain Leader Selection}

After performing clustering the $\mathrm{CH}$ selection step is take place. Select $k$ cluster head from $N$ node, these chain leaders become the leaders in their colonies to control the transmission of data to BS. The proposed method for chain leader selection is based on weight $W$ allocated to each $\mathrm{SN}$. Each node calculates its distance from the BS use Euclidean distance $d i B S$ :

$$
d_{i B S}=\sqrt{\left(x_{i}-x_{B S}\right)^{2}+\left(y_{i}-y_{B S}\right)^{2}}
$$

Where $x_{i}, y_{i}, x_{B S}$ and $y_{B S}$ is the $\mathrm{x}$ and $\mathrm{y}$ coordinate of SNs and BS respectively. The weights are calculated by dividing the nodes residual energy on its distance from BS as following equation:

$W_{i}=E_{i} / d_{i B S}$

Where $E_{i}$ the remaining energy of sensor node $i$ and calculated as following:

$E_{i}=E_{0}-E_{t}$

Where $E_{0}$ initial energy of each $\mathrm{SN}, E_{t}$ is sum of energy that $\mathrm{SN}$ deplete in transmission, reception and aggregation of data. For each cluster, the chain leader is selected by comparing the weights of all the SNs. The node with the maximum weight is chosen as a chain leader.

\section{Step ( $\left.{ }^{\bullet}\right)$ : Chain Building}

In this step the multiple chains building in direction of BS each chain consist one leader, which is last and nearest SN to BS. The single chain building is same as PEGASIS. The proposal is BS transmits hello packet to all nodes in a sensor network collect information about the position, nodes ID and distance. In each cluster, BS finds the farthest $\mathrm{SN}$ from the chain leader node. The farthest node is called as the start SN. Start node finds its nearest node, each node finds the distance from nearest not connected node. Each node $i$ in chain act as a parent to node $(i-1)$, and receives data from it. If one of the nodes in the chains deplete its energy the nodes is bypassed in next rounds and constricting new chain.

\section{Step (6): Energy consumption to proposed algorithm}

Every SN receives data from preceding adjacent, aggregates with its own data, and transfers to the next adjacent in the chain until all data are transferred to the chain leader node, the radio model used described in the Fig. (4).

The free space propagation model is used if distance is beneath a threshold distance value else multi-path fading channel model is used. The energy dissipated in transmitter to transmit $B$-bit packet is given by

$$
E_{T x}(B, d)= \begin{cases}B E_{\text {elec }}+B \epsilon_{f s} d^{2} & d<d_{0} \\ B E_{\text {elec }}+B \epsilon_{m p} d^{4} & d \geq d_{0}\end{cases}
$$

Where threshold distance value $d_{0}$ calculated by following equation:

$$
d_{0}=\sqrt{\epsilon_{f s} / \epsilon_{m p}}
$$

$E_{\text {elec }}$ energy depleted in transceiver part to send and receive one bit, $\epsilon_{f s}$ and $\epsilon_{m p}$ energy depleted in free and multi-path channel model and $d$ distance from node $i$ to node $i+1$.To receive this $B$-bit packet, the radio expends:

$$
\begin{aligned}
& E_{R}(B)=E_{\text {elec }} \times B \text {.............................. (16) } \\
& \quad E_{\text {Aggregation }} \text { energy depleted into data } \\
& \text { aggregation. }
\end{aligned}
$$

$$
E_{\text {Aggregation }}=E_{D A} \times B
$$




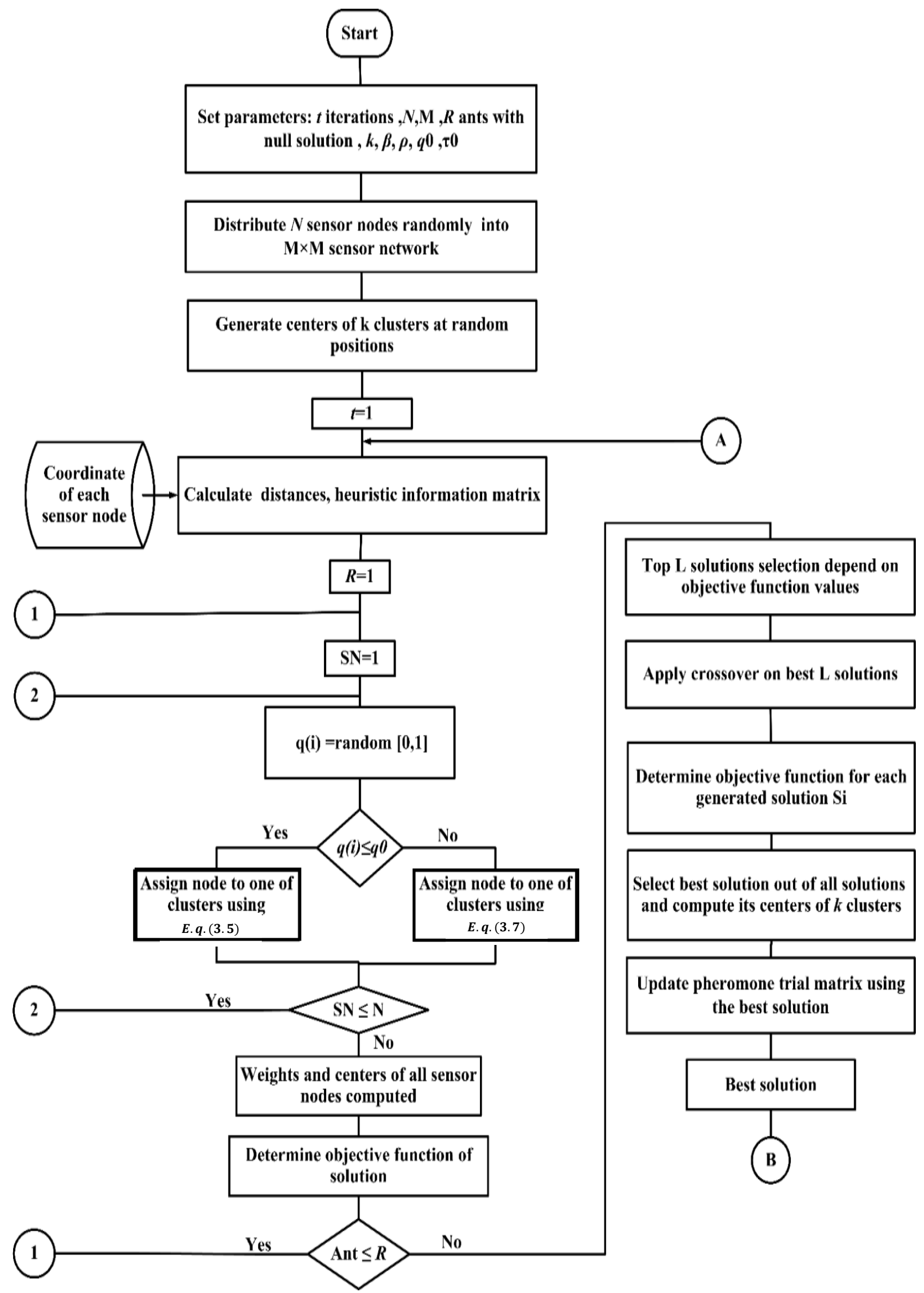

Fig.(5): Flow chart of ACO-PEGASIS part 1. 


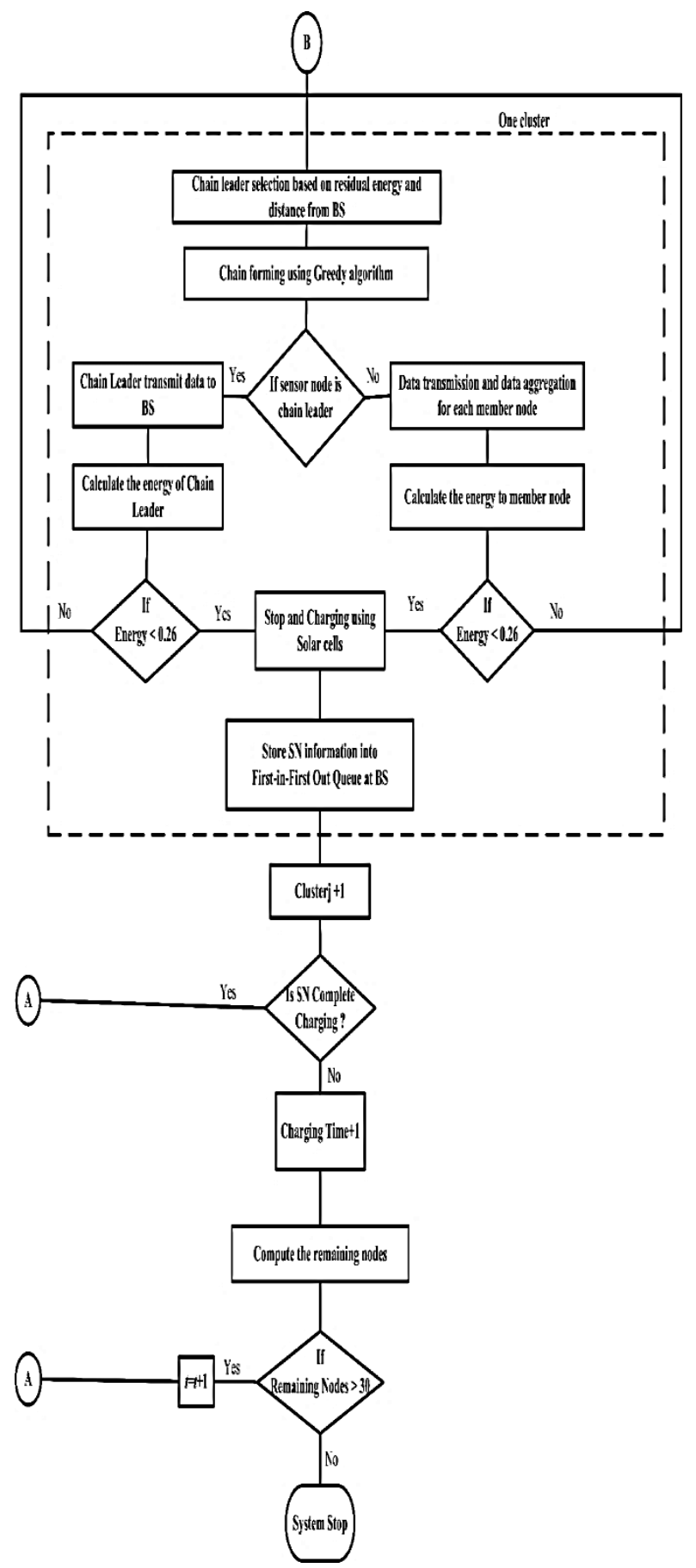

Fig.(6): Flow chart of ACO-PEGASIS part 2.

\section{Results and Discussion}

In order to study the proposed approach, by using MATLAB to simulate different scenarios of 100 sensor nodes deployed uniformly inside a layout of 100x100 square meters. $5 \%$ of the sensor nodes are selected to be chain leaders. The initial energy for each sensor node was set to be 0.5 joule. It is worth to note that the initial energy for the rechargeable batteries scenario was used to recharge sensors to this value. Table (1) summarized the simulation parameters.
Table (1)

Simulation parameters used.

\begin{tabular}{|c|c|}
\hline Parameter & Value \\
\hline \hline Simulation area & $100 \times 100 \mathrm{~m}^{2}$ \\
\hline Number of clusters $(\mathrm{k})$ & 5 \\
\hline \hline Sensors number & 100 \\
\hline Initial energy of Nodes $\left(\mathrm{E}_{0}\right)$ & $0.5 \mathrm{~J}$ \\
\hline \hline Data packet size & $2000 \mathrm{bits} / \mathrm{Packet}$ \\
\hline \hline $\mathrm{E}_{\text {elec }}$ & $50 \mathrm{~nJ} / \mathrm{bit}$ \\
\hline$\epsilon_{\mathrm{fs}}$ & $10 \mathrm{pJ} / \mathrm{bit} / \mathrm{m}^{2}$ \\
\hline$\epsilon_{\mathrm{mp}}$ & $0.0013 \mathrm{pJ} / \mathrm{bit} / \mathrm{m}^{4}$ \\
\hline \hline EDA & $5 \mathrm{~nJ} / \mathrm{bit} / \mathrm{packet}$ \\
\hline \hline Charge threshold & $0.26 \mathrm{~J}$ \\
\hline \hline Recharge time & $2 \mathrm{days}(48$ hours $)$ \\
\hline \hline Threshold node die & 3 times \\
\hline \hline$q_{0}, \tau_{i, j}, \beta, \mathrm{L}, \rho$ for ACO & $0.9,0.01,2,2,0.1$ \\
\hline \hline
\end{tabular}

In order to perform the simulations for proposed algorithms. The following assumptions are made:

- All the SNs in the network are homogeneous and energy constrained, the SNs are stationary.

- The BS is immobile and has no energy constrained.

- SN can measure distances roughly based on the received signal strength.

- All packets that are transmitted at the same size.

- Nodes always have data to send.

- All SNs are sensing the surroundings at a same rate.

- The radio channel is symmetric such that energy necessary for transmitting a packet from a SN (A) to another SN (B) is the similar to the energy required for transmitting a packet from the SN (B) to the SN (A).

- The effect caused by signal collision and interference in the wireless channel is ignored.

- All sensor nodes can be recharged using solar cell.

The performance of the proposed modifications can be evaluated by the following metrics are calculated [10][11]: 
1. Network lifespan: interval from the beginning of WSN work till the expiry of last alive SN or system stop.

2. Stability and instability period: Stability interval from the beginning of WSN operation till the expiry of the first SN. Instability period its time period where SNs start dying till the last alive $\mathrm{SN}$ or system stop. This period should be kept as small as possible.

The Total Residual Energy: It represents the total residual energy of all SNs in the network per round its calculated by the following equation:

$E_{\text {total }}=\sum_{i=1}^{N} E_{c}$

Where Ec current energy level of a $\mathrm{SN}$ and $\mathrm{N}$ number of SNs in the simulation.

The homogeneous network after the chains building for ACO-PEGASIS shows in Fig (7) after 100 round. The proposed algorithms divided the sensor network in to five clusters with unequal size of each cluster.

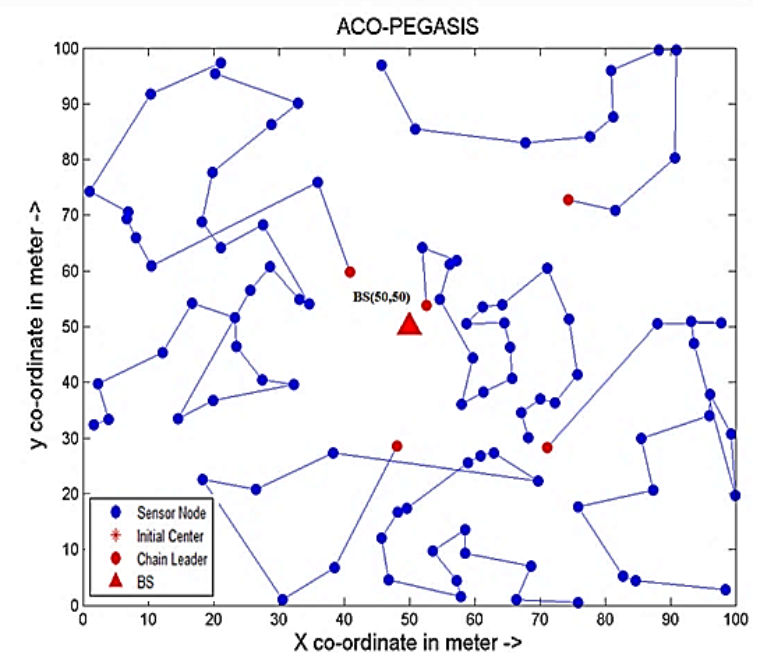

Fig.(7): Chains formed by ACO-PEGASIS.

We compare ACO-PEGASIS with original PEGASIS. We perform simulation for 5000 rounds. Fig.(8) represent the network lifetime for both algorithms. Comparison shows that network lifetime and stability period of ACOPEGASIS is better than that of PEGASIS. Which are approximately 4791 rounds and 4326 rounds respectively. Unlike PEGASIS, ACO-PEGASIS has shorter instability period which is about 465 rounds because energy dissemination is done efficiently. This shows that our proposed algorithm is about $111.49 \%$ better in stability period. From this graph we see that ACO-PEGASIS guarantees about 83.70399 in network lifetime.

The nodes are termed as dead, if they charged for specific charge threshold they drop transmitting or receiving abilities. Fig.(9) shows the residual energy for the proposed algorithm in comparison with original PEGASIS.

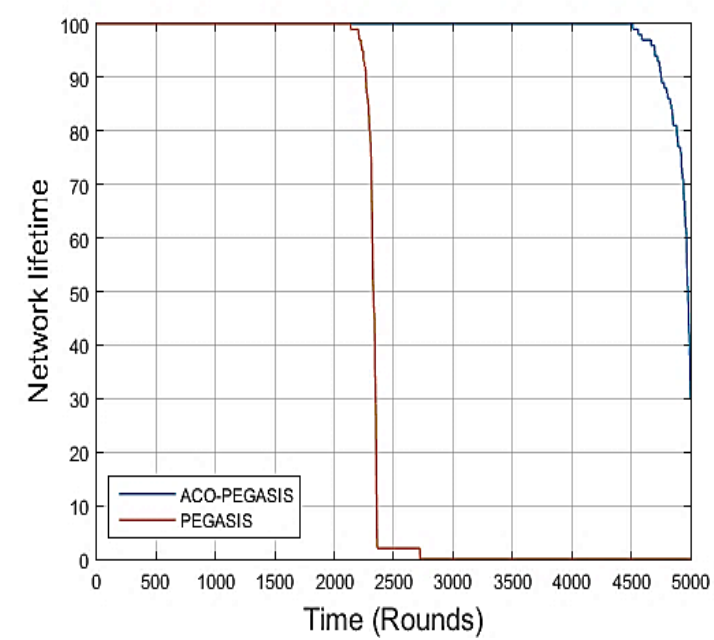

Fig.(8): Network lifetime.

\section{Conclusions}

In this paper, we modify PEGASIS to multi-chain model with using rechargeable sensors to maximize the network lifetime. Our considerations are supportive in diminishing the delay in data delivery and distances between the connected sensors through smaller chains. ACO-PEGASIS lessens the load on the chain leaders. As for future directions, we are striving to get much better sink mobility specifically toward chain leaders of chains in WSN. Evaluation the proposed algorithms by using NS2 program to make better understanding to result analysis. 


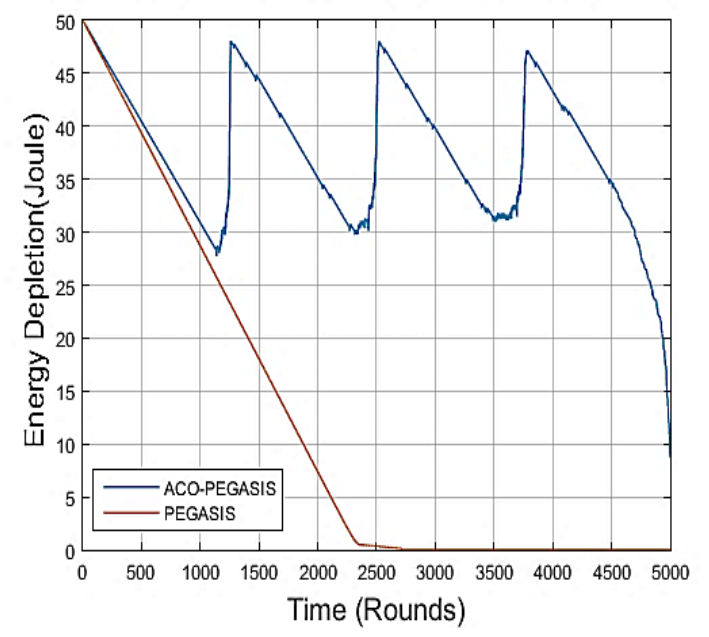

Fig.( (9): Total residual energy.

\section{References}

[1] Chong C. Y. and Kumar S. P., "Sensor Networks: Evolution, Opportunities, and Challenges", IEEE, 91(8), 1247, 2003.

[2] Banu S. and Dhanasekaran R., "A New Multipath Routing Approach for Energy Efficiency in Wireless Sensor Networks", IJCA, 55(11), 24, 2012

[3] Chawla H. and Verma P., "Balanced K Means Based Clustering Algorithm for Energy Efficient in Wireless Sensor Networks", IJARECE, 3(11), 1540, 2014.

[4] Jung S., Han Y. and Chung T., "The Concentric Clustering Scheme for Efficient Energy Consumption in the PEGASIS", ICACT, 260-265, 2007.

[5] Jafri M. R., Javaid N., Javaid A. and Khan Z. A., "Maximizing the Lifetime of MultiChain PEGASIS Using Sink Mobility", CIIT, 21(9) 1283-1289, 2013.

[6] Selem E., Khedr A. M. and Nassar H., "MPEGASIS: Energy Efficient Pegasis for Mobile Wireless Sensor Networks", ECS, 37(6), 33-43, 2013.

[7] Tan N. D. and Viet N. D., "SSTBC: Sleep Scheduled and Tree-Based Clustering Routing Protocol for Energy-Efficient in Wireless Sensor Networks", IEEE RIVF, 180-185, 2015.

[8] P. S. Shelokar, V. K. Jayaraman and B. D. Kulkarni, "An ant colony approach for clustering", ACA, 509 (2), 187-195, 2004.

[9] ZHAO B. J., "An Ant Colony Clustering Algorithm", IEEE, 3933-3938, 2007.

[10] Chunawale A. and Sirsikar S., "Minimization of Average Energy
Consumption to Prolong Lifetime of Wireless Sensor Network", IEEE GCWCN, 246, 2014.

[11] Khan Z. A. and Sampalli S., "AZRLEACH: An Energy Efficient Routing Protocol for Wireless Sensor Networks", IJCNS, 5(11), 792, 2012.

الخلاصة

PEGASIS

نموذج متعدد سلسلة باستخدام التجمعات و أجهزة الاسنشعار القابلة لإعادة الثحن لتحقيق أقصى قدر من عمر الثبكة. الغرض التقليل من التأخير في تسليم البيانات والمسافات بين أجهزة استشعار متصلة من خلال سلاسل أصغر -ACO. PEGASIS للاتجاهات المستقبلية، ونحن نسعى للحصول على أفضل بكثير التقل بالوعة تحديدا نحو القادة سلسلة من سلاسل في WSN .NS2(network simulator 2) 